\title{
An Assessment Model for the State of Organizational Readiness Inservice Oriented architecture Implementation Based on Fuzzy Logic
}

\author{
Akram Hedayati ${ }^{1}$, Babak Shirazi ${ }^{2}$, Hamed Fazlollahtabar ${ }^{3, *}$ \\ ${ }^{1}$ Department of Information Technology Engineering, Mazandaran University of Science and Technology, Babol, Iran \\ ${ }^{2}$ Department of Industrial Engineering, Mazandaran University of Science and Technology, Babol, Iran \\ ${ }^{3}$ Faculty of Industrial Engineering, Iran University of Science and Technology, Tehran, Iran \\ *Corresponding author's email: hfazl@iust.ac.ir
}

Copyright (C) 2014 Horizon Research Publishing All rights reserved.

\begin{abstract}
Growth changes in organizations and on the other hand strong need to utilization of information and communication technology possibly implied applying changes in organization in accordance with changes in functionality. What's more, information technology structure, hardware, software and information infrastructure have to be synchronized. One of the most important approaches leading to this goal are service oriented architecture.Service oriented architecture (SOA) is a style of information systems architecture supporting loose coupling of services for flexibility and interoperability of systems and is independent of the technology. The most important achievement of creating service oriented architecture is the need for increasing flexibility, speed and agility. Consequently, it changes in the structure of its organization. According to broad scope of enterprise architecture projects, it is necessary before implementation and the related major cost, organization would be notified of its readiness to get right decisions, develop appropriate strategies and adopt new approach.In this paper, we propose a model for evaluating organizational readiness in order to implement SOA with the use of Mamdani fuzzy logic. According to the results of this assessment method, we can decide more precisely whether an organization is ready to adopt SOA or not.By using this assessment model, organizations can realize their strengths and weaknesses identify their improvement areas and thus increase their readiness. Consequently this model is tested by means of a case study in Tehran municipality ICT organization.
\end{abstract}

Keywords Service Oriented Architecture (SOA); Organizational Readiness; Fuzzy Logic

\section{Introduction}

Increasing growth changes in organizations, makes organizations get more complex continuously. Without using of information technology, many problems occur in order to achieve the organization's goals. Planning to achieve these organizational objectives needs a real understanding of the current situation which is obtained by readiness assessment measures; on the other hand in transferring processes and data changes to its applications and related issues, we should act flexible.

SOA is a relatively young phenomenon. This creates a strong need for more knowledge about SOA and standardized SOA implementation prerequisite [1]. The concept of service oriented architecture provides organizations the ability to reuse their existing assets and contributes to a higher level of organizational agility and flexibility. Organization can implement SOA rightly, so all processes and services are quite flexible and quickly. Therefore, it can be created and configured based on the business requirements but no needing the professional or technical personnel. This agile view is according to growing needs of changing business environment and constraints of organizational and technical infrastructure too.To implement the new paradigm, in order to avoid spending too much time and cost due to lots of attention or lack of consideration of organizational factors that ultimately might lead to failure and bankruptcy of project implementation, various perspectives including financial, time, human factor, and technical and so on have to be evaluated before implementation so that the current situation would be clear and make decision about adopting new paradigm.

This paper elaborates on and categorizes these measures that help decision makers (1) know the current organizations' readiness and decide more precisely whether or not to implement SOA (2) select the measures that fit in with their objectives (3) identify the defects and flaws aspects, and improve them by using some provided suggestions. Organizational decisions often are taken place in an uncertain environment by unclear and ambiguous criteria and insufficient information. If the uncertainty (fuzziness) is 
not considered in decision making, the results can be misleading. In order to promote, improve results, precise assessment and efficient assessment processes, fuzzy sets and fuzzy logic are used. Fuzzy logic translates human understanding and natural language to semi-mathematic algorithms. A model which is based on fuzzy logic includes fuzzification, inference and is defuzzification.

In the second section, we investigate previous assessment readiness model and critical success factor for a successful SOA implementation. In the third section the research methodology will be explained and the factors that influence SOA will be identified. Furthermore, in the fourth section we will explain the procedure of making model by fuzzy logic. Lastly, considering one of Iranian company as a case to study, the obtained results are reported and some future research directions are outlined.

\section{Background}

The latest architectural revolution to strike IT is SOA [2]. But there is no specific definition for service-oriented architecture (SOA), but most agree that this architecture is related to the lower layers of organization. It is based on technology and its impact on both business and technology domain is obvious[3].SOA is a framework for integrating service-oriented business processes and supports them by standard and secure components which are called service are reusable and loose coupling to cover all business process changes[4].

SOA readiness assessment research should be conceptualized such a model so that consider all relevant and effective features and because this context is one of a subset of large-scale readiness assessment IT projects [5].Since now studies show extensive literature on general readiness assessmentIT-projects in organizations.Here, we just review them quickly. For instance, we can refer to APEC model [6],WISTA model[7], and BTOB-EC model [8] to assess e-commerce readiness,Al-omari model[9] to to assess e-government readiness, Hanafizade model [10], CSPP model[11] and CIDCM model [12] to assess ICT readiness, Tohoca model [13] to assess e-learning readiness, OITIM model [14], Fehrenbach model [15], Mirzaei model [16]to assess information systems (IS)readiness. Also Shanaka et al. provided a taxonomy of CSFs for Eenterprise Resource Planning (ERP) implementation [17]. Rosalie et al. provided an assessment model for customer relationship management $(\mathrm{CRM}))$ [18]. Anjariny et al. proposed a model for business intelligent systems readiness assessment model [19].

SOA is a relatively young phenomenon. This creates a strong need for more knowledge about SOA and standardized SOA implementation prerequisite[20].Conway has defined SOA readiness as being when an organisation understands what SOA is, and recognizes the problems, costs, benefits and limitations associated with a SOA approach [21]. In literature on readiness assessment of SOA implementation, the critical success factors (CSF), SOA maturity, and governance issues have been pointed. CSFs are defined as a small set of goals or prerequisites if addressed appropriately will significantly improve the chances of project success [22]. CSFs can guide practitioners on where they should focus attention and resources when implementing enterprise projects[22].SOA governance means get to assure about concepts, principles and SOA rules and distributed management and creating services based on business goals[23].This knowledge defines which specific issues are important at what time.

Legner et al. identified three major activities which are important for successful SOA adoption: introduction of organisational roles and governance processes; creation of architectural guidelines; and use of SOA for infrastructure projects [24]. Vegter focused on reusability of services, reducing complexity of SOA and some activities of SOA governance as the main CSFs in a SOA project [25]. Lawler et al., also identified the CSFs that contribute to effective planning and effective management of SOA from technical, procedural and business perspectives; they found that business factors are the most important in managing SOA strategy [26]. Lee et al. used both SOA maturity and CSFs in a study of SOA implementation in Korean companies. Their CSFs included awareness factors, SOA strategy, organisational management, technology infrastructure, project management, governance and technology infrastructure and totally considered 20 indicators [27]. Another study byAbdul Mananet al. proposed an Enterprise SOA readiness framework suitable for public sector organisations. The methodology used from maturity models and CSF for a SOA implementation readiness and contains 6 dimensions as follows: SOA Awareness, SOA strategy, IT governance, SOA governance process, SOA infrastructure and technology, SOA competency [22]. Other researchers focused on governance as the critical issue for Enterprise SOA adoption. Most SOA governance frameworks are extensions of IT governance frameworks, which define governance structures, governance processes and behaviour mechanisms, which are themselves still immature concepts in the literature [28].

Studies show that there is not any exact and accurate assessment model for successful (SOA) implementation and organizations cannot assess their readiness for successful implementation. In addition, lacks a proper reference model for evaluation. Also, a clear definition of what constitutes SOA readiness and empirical research on SOA readiness is required.

\section{Research Methodology}

This study aims to develop the state of knowledge and also is a applicable study so that can be used for assessment of readiness of Iranian organizations toward successful SOA implementation. Based on the context and methods, this paper is a descriptive-survey category because it aims to recognize the factors affecting organizational readiness by 
referring to a specific community.

For validation, assign the weights of indices and determine fuzzy rules, our expert community includes persons who are expert on information technology (IT) and service-oriented architecture field.Based on assumptions and by considering fuzzy approach, a questionnaire has been designed in one section and distributed among experts. The feedback and comments collected from experts have been used to make necessary adjustments to satisfy potential needs of organization. Mamdani fuzzy logic is used for proposing this model. Construction is comprised of four main components: fuzzification, determine rules, inference, and defuzzification. Fuzzy modelling has been performed with Matlab software. The overview of the flowchart is shown in Figure 1.

Achieving conceptual model for readiness assessment of SOA has two stages as follows: first of all, the main readiness dimensions for readiness assessment is conceptualized and have been discovered; second, to measure more precisely, each of those dimensions are divided in to indicators (we call them sub-dimension). Multifaceted sub-dimensions caused that we consider some sub-indicators for them. By summarization and classification studies, now we have a specific framework and general conceptual model for assessment of SOA readiness in organizational scale toward design of a fuzzy expert system. Conceptual model is expressed in the following table. This model covers different aspects of assessment of SOA readiness and contains 3 overall dimensions, 8 indicators and 30 sub-indicators.

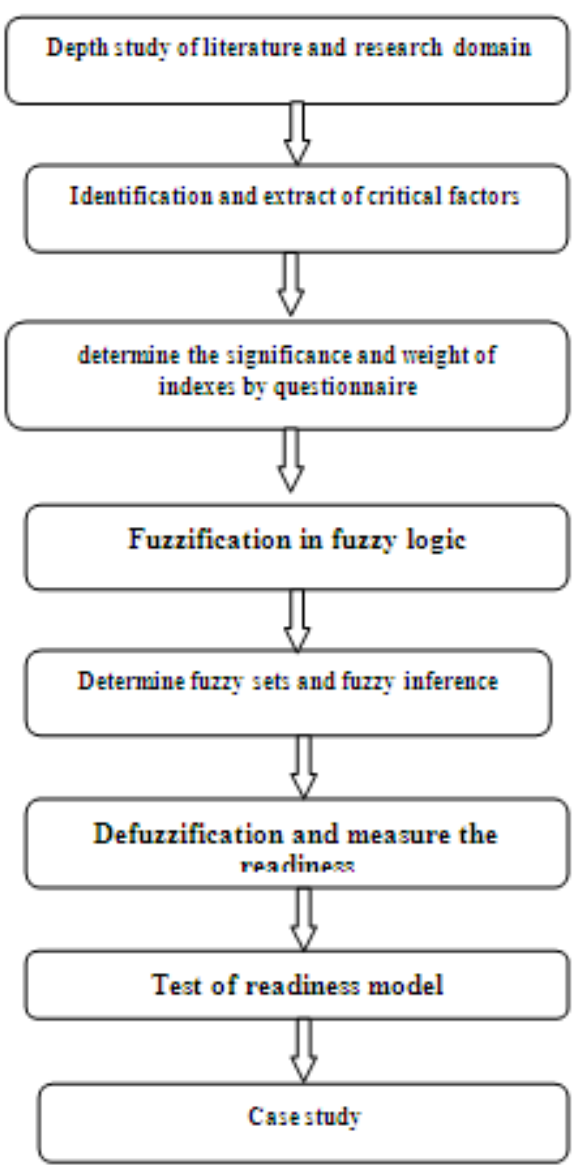

Figure1. Overall view of flowchart 
An Assessment Model for the State of Organizational Readiness Inservice Oriented architecture Implementation Based on Fuzzy Logic

Table1. Conceptual model

\begin{tabular}{|c|c|c|c|}
\hline $\begin{array}{c}\text { Main } \\
\text { dimensions }\end{array}$ & $\begin{array}{l}\text { Sub-dimension } \\
\text { (indicators) }\end{array}$ & Number & Sub indicators \\
\hline \multirow{12}{*}{$\begin{array}{l}\text { Business } \\
\text { readiness } \\
\text { dimension }\end{array}$} & \multirow{2}{*}{$\begin{array}{l}\text { Readiness of organizational } \\
\text { Culture }\end{array}$} & 1 & Creating culture of innovative and improvement organizational culture \\
\hline & & 2 & Definite model of roles and responsibilities \\
\hline & \multirow{4}{*}{$\begin{array}{l}\text { Readiness of organizational } \\
\text { Management }\end{array}$} & 1 & Service management and service support \\
\hline & & 2 & $\begin{array}{c}\text { Management of strategy, costs, risks ,expectations and organizational } \\
\text { changes }\end{array}$ \\
\hline & & 3 & Strengthening business service oriented design process \\
\hline & & 4 & $\begin{array}{l}\text { Information management } \\
\text { And information systems } \\
\text { Portfolio } \\
\end{array}$ \\
\hline & \multirow{3}{*}{$\begin{array}{l}\text { Readiness of } \\
\text { SOA awareness }\end{array}$} & 1 & Clear and deep understanding of SOA concept in organization \\
\hline & & 2 & Sharing experiences of successful SOA with business users \\
\hline & & 3 & $\begin{array}{l}\text { SOA training and raising the level of SOA knowledge and (in proportion with } \\
\text { the level of education and organizational knowledge( }\end{array}$ \\
\hline & \multirow{3}{*}{$\begin{array}{l}\text { Readiness of } \\
\text { SOA strategy }\end{array}$} & 1 & Alignment of SOA strategy and it strategy, \\
\hline & & 2 & SOA strategy supports organizational values \\
\hline & & 3 & Clear SOA project roadmap and clear vision and goal-setting \\
\hline \multirow{10}{*}{$\begin{array}{l}\text { Governance } \\
\text { readiness } \\
\text { dimension }\end{array}$} & \multirow{5}{*}{ Readiness of it governance } & 1 & $\begin{array}{l}\text { It leadership committee supports it governance and } \\
\text { Executive leadership technologies }\end{array}$ \\
\hline & & 2 & Budget supports \\
\hline & & 3 & $\begin{array}{c}\text { Collaboration and } \\
\text { Sharing knowledge between business units \& it.And } \\
\text { Fostering a partnership culture }\end{array}$ \\
\hline & & 4 & $\begin{array}{l}\text { Experts for determine policies and service orientation and active in SOA } \\
\text { decision field and SOA related problems }\end{array}$ \\
\hline & & 5 & It understanding of expected business benefits and SOA projects delivery \\
\hline & \multirow{5}{*}{$\begin{array}{l}\text { Readiness of } \\
\text { SOA governance }\end{array}$} & 1 & \begin{tabular}{ll} 
& \multicolumn{2}{c}{ SOA } \\
Center & of excellence \\
\end{tabular} \\
\hline & & 2 & $\begin{array}{c}\text { Clear conventions ,policies ,roles, responsibilities and business process } \\
\text { reengineering and continuous improvement }\end{array}$ \\
\hline & & 3 & Process and service policies, deployment techniques and interactions \\
\hline & & 4 & Support and executive business leadership \\
\hline & & 5 & $\begin{array}{l}\text { Step by step evolution planning with consideration of current organization } \\
\text { capacity }\end{array}$ \\
\hline & \multirow{4}{*}{$\begin{array}{l}\text { Readiness of technology } \\
\text { infrastructure }\end{array}$} & 1 & $\begin{array}{cc} & \text { Xml } \\
& \text { SOA standard technology including } \\
\text { Management standards } \\
\text {,web-services, } & \text { security, user interface, generation and develop services, } \\
\text { messaging, } \text { xml }\end{array}$ \\
\hline \multirow{7}{*}{$\begin{array}{l}\text { Technical } \\
\text { readiness } \\
\text { dimension }\end{array}$} & & 2 & $\begin{array}{c}\text { Management business process reengineering software, data } \\
\text { tools ,middleware, platform of special tools of developed companies, } \\
\text { modeling information and processes }\end{array}$ \\
\hline & & 3 & Organizational architecture management system \\
\hline & & 4 & $\begin{array}{c}\text { Definition of technology domain ,development methods, and make common } \\
\text { and right infrastructure } \\
\text { (based on previous it investment) } \\
\text { And deploying process and services techniques }\end{array}$ \\
\hline & \multirow{2}{*}{$\begin{array}{l}\text { Readiness of facilitating } \\
\text { reusability of services }\end{array}$} & 1 & $\begin{array}{l}\text { Core function in quality services section to control granularity and acceptable } \\
\text { level of reusability }\end{array}$ \\
\hline & & 2 & $\begin{array}{l}\text { Adjust interaction policies, development services and evaluate the expected } \\
\text { service delivery }\end{array}$ \\
\hline & & 3 & $\begin{array}{c}\text { Creating } \\
\text { An environment with culture that encourages the development of reusable } \\
\text { software and services }\end{array}$ \\
\hline & & 4 & Focus on reduce complexities \\
\hline
\end{tabular}




\subsection{Fuzzification}

A fuzzifier converts crisp input to fuzzy data [29]. At this stage, facts are defined based on fuzzy systems then inputs and outputs are defined. Fuzzification phase consists of two steps: (1) after assigning the weights of sub-indicators and indicators, fuzzifying input variables and considering fuzzy membership function (2) fuzzifying output variables and considering fuzzy membership function. Extracted effective indicators and sub-indicators are as fuzzy expert system input and final amount of assessment of organizational readiness to SOA implementation is as a output of system. Weighing system inputs are calculated by the Minkowsksy formula.Defined linguistic variables of factors have been illustrated inFigure2 ( $a$ and $b$ ), in which fuzzification inputs are in triangular fuzzy numbers and their range is between 0 and 100. In the current study, due to more validity, frequency of use and the ease of calculation, triangular membership functions are used for fuzzification. Certainly, the other shapes of membership functions can be used instead.

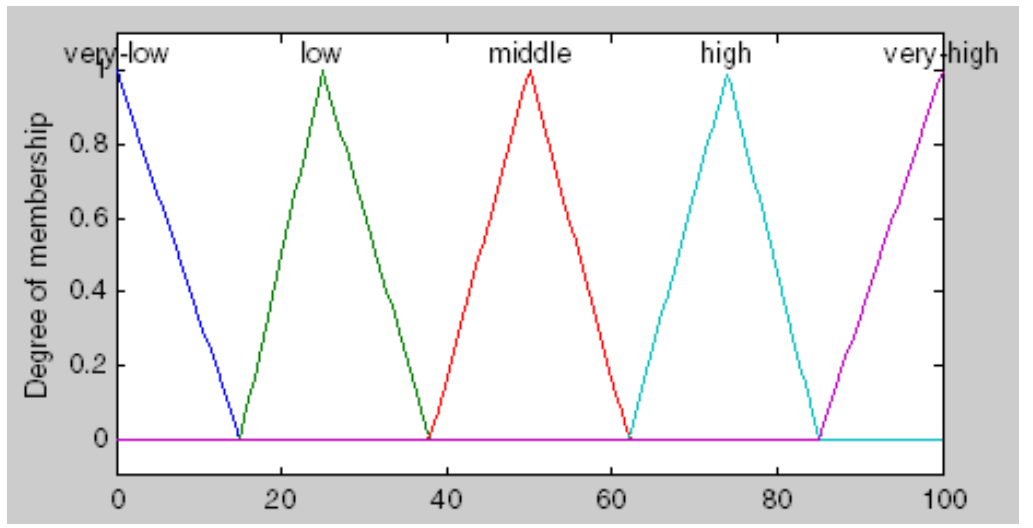

Figure 2 (a). Membership function for linguistic values of input

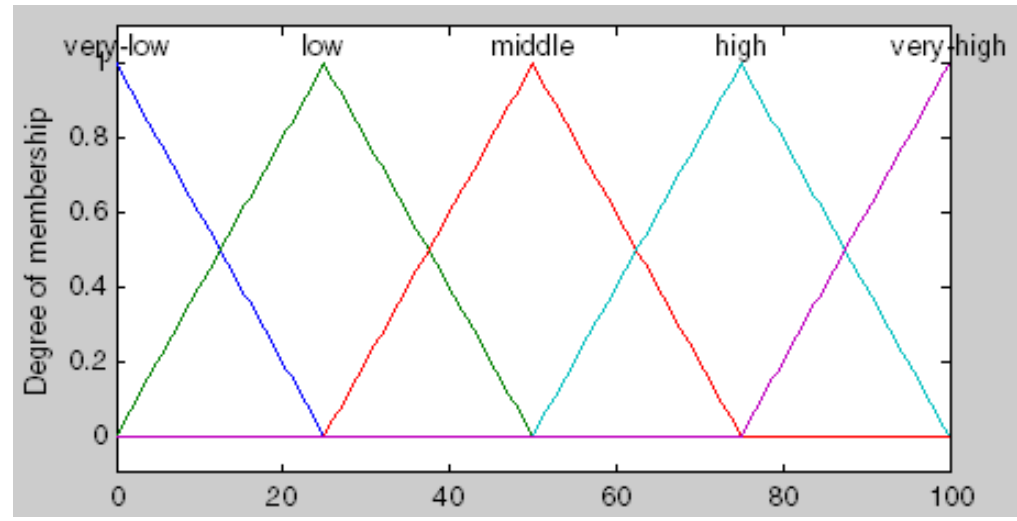

Figure 2 (b). Membership function for linguistic values of output

\subsection{Determine Fuzzy Rule Sets}

At this stage, the rule base is created. According to the terms of the values assigned to each variable and also the fuzzy if-then rules of the system, its behavior is evaluated. Dimensions and sub-dimensions of model are shown in Figure 3. To write the rules, according to the information obtained from experts, rules are written for each sub-dimension separately, then the outputs of these rules are combined in second level, and make new rules that determine the readiness of organization in three main dimension (business, governance, technical readiness). Then in the third level, according to different combination of organization readiness and their correlation at these 3 main dimensions, final amount of assessment of organizational readiness to SOA implementation is calculated.

According to Mamdani principles, the written rules are multi-input single-output (miso). As we see in Fig.3, each triangle has been used to show the existence of a set of rules (a fuzzy inference engine). An inference engine has been designed for each triangle, totally 12 engines. According to calculated weights, input variables, are not in the same priority but the weights of all rules is equal to1.Totally 241 rules has been designed.It is not necessary to increase the number of rules in rule base of fuzzy expert system. The rules with high degree of importance should be selected for the speed and accuracy of the fuzzy expert system is not increased with the number of rules necessarily. 


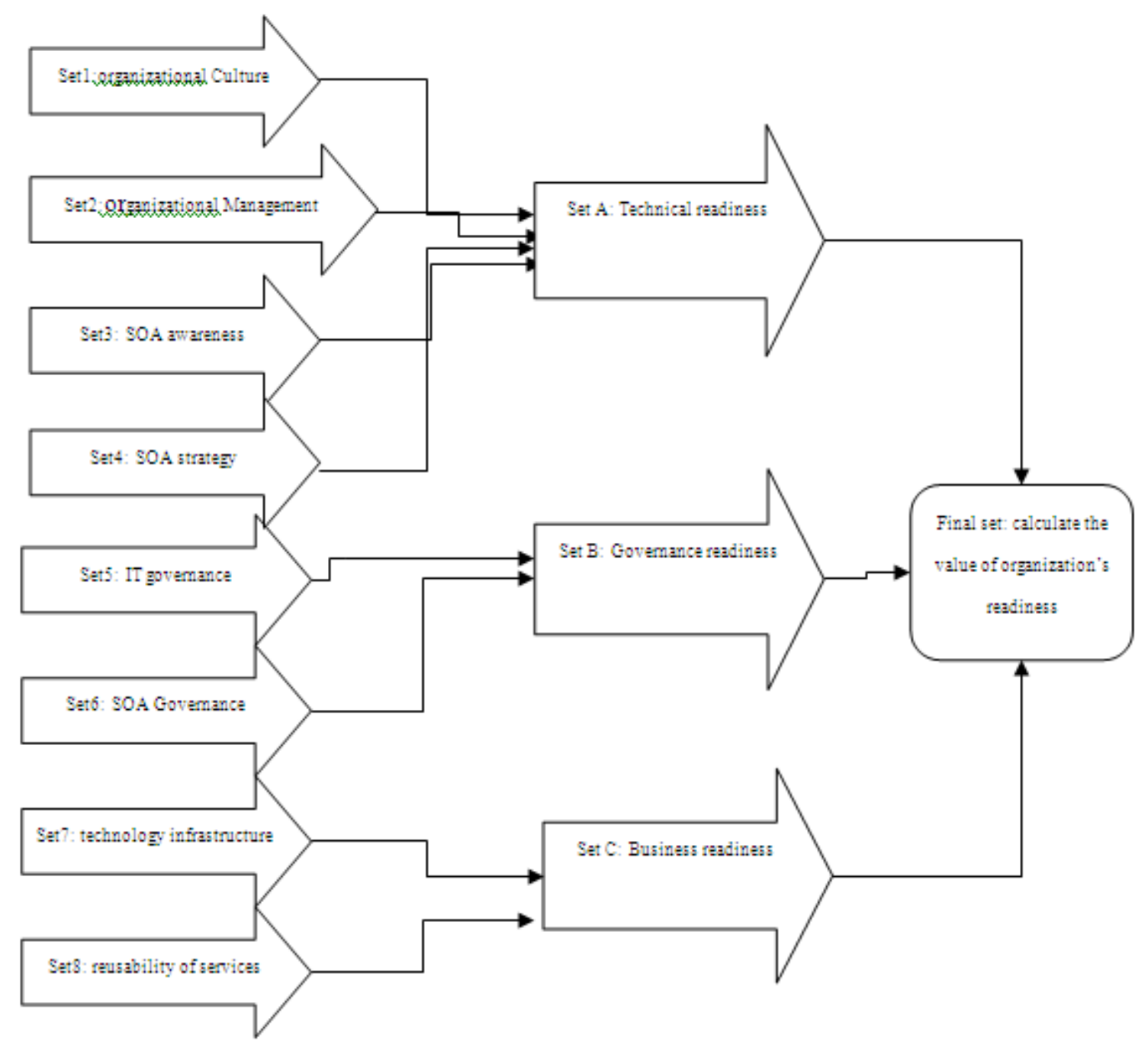

Figure 3. Diagram of designed fuzzy inference system

\subsection{Fuzzy Inference}

Fuzzy inference is the important processing step in fuzzy the expert system and is done by considering rules. According to Mamdani principles, the fuzzy inference process follows "min-max" inference and forward chain inference algorithm. Min method is used for and operator and max method is used for or operator. The "and" operator is used for implication and "max" operator for aggregation.

\subsection{Defuzzification}

In order to use the output value in the real-world, fuzzy value must be translated into real values and this process is called defuzzification and it converts fuzzy set to crisp value [29]. There are many methods of defuzzification such as centroid, center of sums, mean of maxima and left-right maxima.Here centroid method is used in model.

Since any designed model due to lack of considering all real world aspects, has some errors. If the error does not excess threshold, it has relative validity otherwise it would be revised. For testing, in each inference system, two input variables are considered constant whereas we increase or decrease the value of other variables. For each different combination, the output is calculated by fuzzy expert system. These different output values form a behavior for output.

After analyzing the behavior, if this behavior which is obtained by fixing some values and varying the rest of values, adapts with literature or expertise of experts, the validity of model is confirmed otherwise the proposed system have to be revised .outputs for various instances of the input factors have been calculated by Matlab software and also have been compared by tactic knowledge of experts and literature, those confirmed the validity of outputs. 


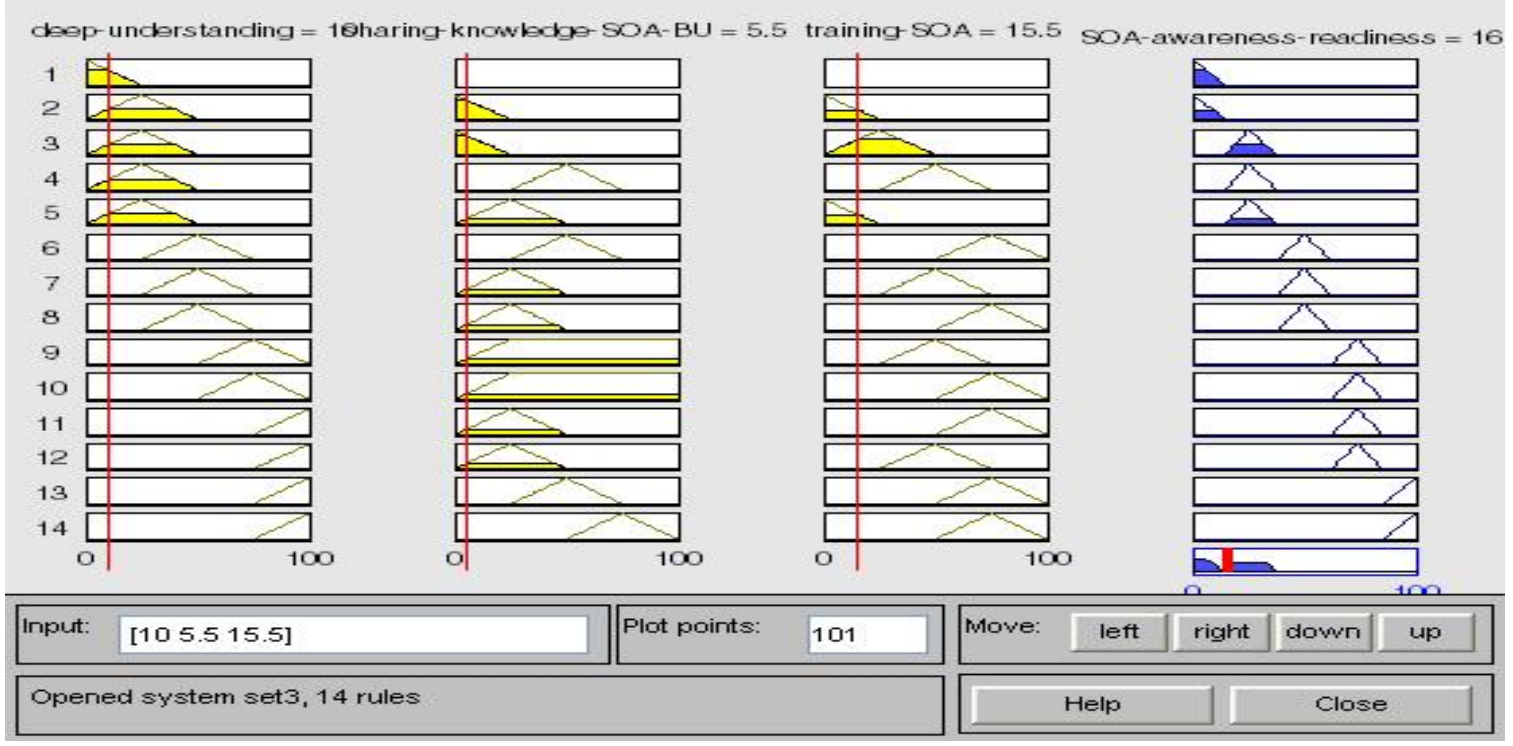

Figure4. Output of inference system of SOA awareness sub-dimension

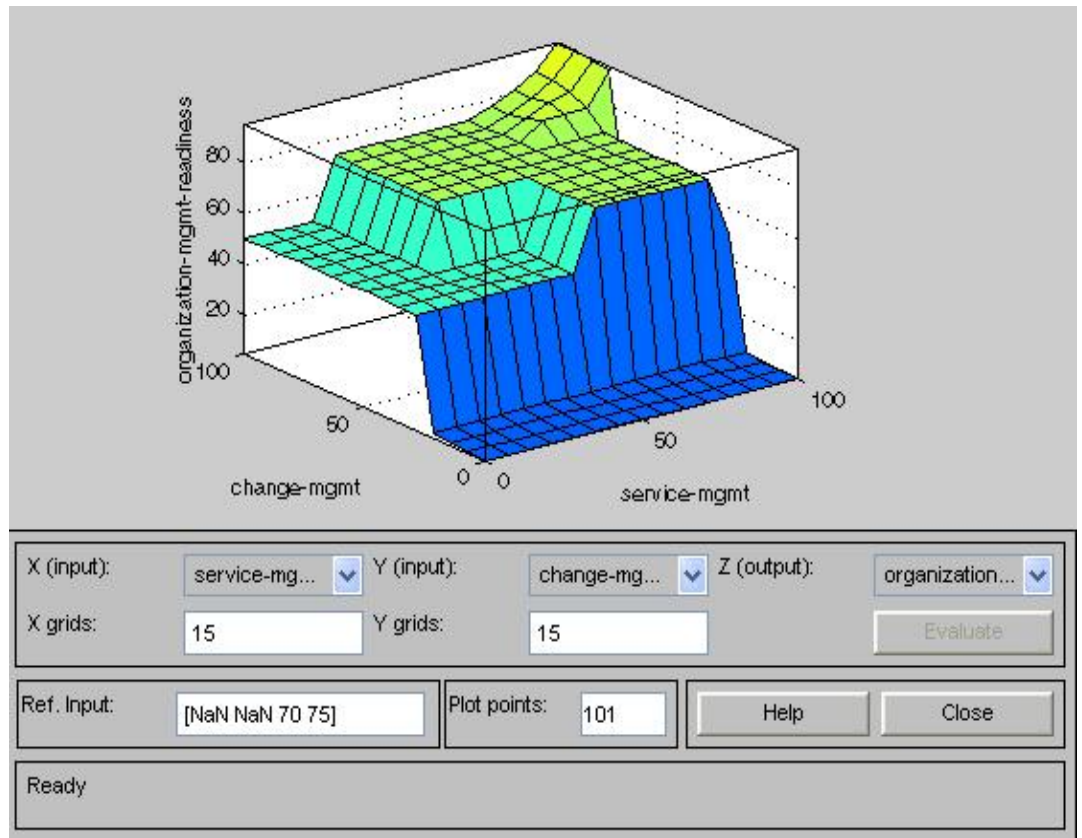

Figure 5. The sensitivity of fuzzy expert system for organizational Management sub-dimension

\section{Case Study}

Specifically, we present a case of study. We selected Tehran municipality ICT organization. The state of organizational readiness for SOA implementation has been evaluated in terms of 30 sub-indicators. The values of expert system inputs have been determined by questionnaire and experts were expected to estimate the current state with number in range of 0 to 100 . At the final step, mean values have been calculated and have been entered as the system input then the final amount has been got the results of using designed model in case study shows that it has very-low readiness at SOA awareness, SOA strategy, technology infrastructure sub-dimensions. Then organization in order to improve its readiness for SOA implementation needs overall review in the corresponding sub-indicators these are as critical dimension of organization. Also it has middle readiness level at SOA governance sub-dimension. By considering the activity domain of organization in it domain, on the other hand, SOA concept is a subset of large-scale readiness assessment IT projects [5], unconsciously some of proposed sub-indicators are the same as in critical factors in IT, these would have high readiness level at organizational management, IT governance, facilitating reusability of services sub-dimensions. The readiness of organizational culture has estimated very considerable and is at very-high 
level. By considering the weights and determined priorities, knowledge of experts and extracted rules, we could estimate state of organizational readiness at 3 main dimensions: business readiness, governance readiness, technical readiness. Results show it has middle readiness level at business readiness and technical readiness dimensions and with little membership degree, has high readiness at governance readiness dimension and finally the state of readiness of whole organization has estimated at middle level analysis show that if the above organization is planning to implement SOA, to avoid failure and increase success, it needs to consider special activities to improve it is current state.

For instance, we see the output of one of the inference systems in the Figure4.

As shown in Figure 5, the sensitive analysis can be done in the rule base to extract the relationship among the effective parameters in dimensions and sub-dimensions. As we see the 3 area, it is about the output changes based on changes in inputs.

\section{Conclusions}

SOA offers a great opportunity to organizations, adding greater value, increases in customer services while reducing cost and reducing wasting time and enhancement of their business. Also enables us to handle rapid business changes with more flexibility and agility in our products. With considering overall and comprehensive view and most effective factors for SOA implementation, in this paper we presented a new model so that organizations can evaluate their state of readiness for successful SOA implementation. After extensive study, we have elicited all critical factors for SOA pre-implementation but just thirty of them which were the most important have been elicited. The opinions of experts have been elicited by questionnaire which made their mind convergent among the huge uncertainty. Studies show that up to now there was no exact and comprehensive readiness assessment model of SOA that consider all related aspect.Not only high-level policy makers need these type of assessment models, surely all managers in organizations and also public and private companies have to be aware of their current readiness state so that by using those models at pre-implementation phase, save large amount of potential expenditure for problem resolution. The main advantages of this proposed model is easy understanding and easy use without needing familiar with statistics and reducing evaluating errors by using fuzzy inference. On the other hand by using this model, organization realizes it's strengths and weaknesses and cause more precise decision-making whether start project or not. Future applications of the model will contribute to refining of the clustering concept in fuzzy logic and also combining neural network with fuzzy expert system at designing model. In addition, we can expand the designed model so that it can evaluate the organizations' readiness for enterprise resource planning (ERP) implementation and SOA implementation simultaneously and this could be a station for future research.

\section{REFERENCES}

[1] Legner, C.Heutschi, R. "SOA adoption in practice - Findings from early SOA implementations", in proceedings of the 15th European Conference on Information Systems, St. Gallen, 2007.

[2] Karascony," 4 Step To SOA",Www.Computerworld.Com , 2006

[3] Jae Choi, K. (Ram) Ramamurthy, "Service-Oriented Architecture and IT-Business Alignment", Proceedings of the International Conference on Industrial Engineering and Operations management, 2011.

[4] Bieberstein, N., Bose, S. and Fiammante, M. "Service-oriented architecture (SOA) compass: business value, planning, and enterprise roadmap", IBM press developerWorks series, Pearson education.2006, 65.

[5] Ericson, J., and Siau, K. 2008. "Critical Success Factors in SOA Implementation," in: Americas Conference onInformation System Implementation

[6] Asian pacific economic corporation (apec),"e-commerce readiness assessment guide"

Auckland:apec[online],2000,availableat:Http://www.schooln etafrica.net/fileadmin/resources/apec_e

[7] The world information technology and services alliance (WITSA), "international survey of electronic commerce", london., www.witsa.org/papers/ecomsurv.pdf, 200012

[8] Norjansalika janom1, mohdshanudin zakaria2," b2b e-commerce readiness assessment indicators based on the critical success factors", international conference on electrical engineering and informatics - ieee,2009,

[9] Al-omari, a and al-omari, h "e-government readiness assessment model", journal ofcomputer science , 2006-page $841-845$

[10] Hanafizadeh, p. Hanafizadeh, m. And khodabakhshi "taxonomy of e-readiness assessmentmeasures", international journal of information management “, 2009, page189-195

[11] Computer systems policy project, "readiness guide for living in the networked world" ,1998, available at: http://www.cspp.org/projects/readiness/

[12] Center for international development and conflict management(CIDCM) at the university of maryland ,"negotiating the net model", available at: www.bsos.und.edu/cidcm/projects/neo.html1998

[13] Machado .c ,"developing an e-readiness model for higher education institutions: results of a focus group study", british journal of educational technology, vol 38 no 1,2007 , page $72-82$

[14] Snyder-halpern. R. "indicators of organizational readiness for clinical information technology/systems innovation: a delphi study", international journal of medical informatics , 2001 
[15] Wild, e.1,fehrenbach, s.n, "assessing organizational readiness and capacity for developing an integrated child health information system",2004

[16] Mirzaei, j. And mosavimadani, f, "proposing a conceptual readiness assessment model of mis/is development in manufacturing companies", international conference of information,process, and knowledge management, ieee,2009

[17] shanaka de soysa and juliannanayakkara ,"readiness for ERP implementation in an organization development of an assessment model”, 2006 ieee, page27-32

[18] Rosalie j. Ocker, susanmudambi, "assessing the readiness of firms for CRM:A literature review and research model", proceedings of the 36th hawaii international conference on system sciences, 2002 ieee.

[19] Ahmed H. Anjariny, Akram M. Zeki," Development of Model for Assessing organizations' Readiness Toward Successful Business Intelligence Systems", Research and Innovation in Information Systems (ICRIIS), 2011 International Conference on, Page(s) 1-6

[20] C. Legner, R. Heutschi, "SOA adoption in practice - Findings from early SOA implementations", in proceedings of the 15th European Conference on Information Systems, St. Gallen, 2007.

[21] Conway, D. 2009. "SOA Readiness - A Perspective." Retrieved 24 June, 2011, from http://www.bpminstitute.org/articles/article/article/soa-readi ness-a-perspective/news-browse/7.html
[22] Mahshitah Abdul Manan, "Enterprise Soa Implementation Readiness: A Case Study In Malaysia", Acis Proceedings, Paper 64, 2011.

[23] Miko Matsumura, BjoernBrauel, Jignesh Shah," Soa Adoption ForDummie" Wiley Publishing, 2009.

[24] Legner, C., and Heutschi, R. 2007. "SOA adoption and in Practice - Findings from early SOA Implementations," in: European Conference on Information Systems

[25] Vegter, W. 2009. "Critical success factors for a SOA implementation A case study in the financial sector " in:11 thTwente Student Conference on IT. Enschede.

[26] Lawler, J.P., Benedict, V., Howell-Barber, H., and Joseph, A. 2009. "Critical Success Factors in the Planning of a Service-Oriented Architecture (SOA) Strategy for Educators and Managers," Information Systems Education Journal, 7(94) (7:94).

[27] Lee, J.H., Shim, H.-J., and Kim, K.K. 2010. "Critical Success Factors in SOA Implementation " InformationSystems Management (27), pp 123-145.

[28] Lee, J., and Lee, C. 2009. "IT Governance-Based IT Strategy and Management: Literature Review and Future Research Directions," in: Information Technology Governance and Service Management: Frameworks and Adaptations

[29] Zadeh, L.A.: Fuzzy sets. Information and Control 8, 338-353 (1965) 